\title{
Foley balloons' tamponade is an effective method for controlling massive pelvic bleeding during colorectal
} surgery

\author{
Tzu-Chi Hsu
}

Division of Colon and Rectal Surgery, Department of Surgery, Mackay Memorial Hospital, Mackay Medical College, Taipei, Taiwan

Purpose: Hemostasis can be achieved by various methods, but it can be difficult to stop active bleeding in the pelvis. An effective method is described to stop massive active bleeding in the pelvis during colorectal surgery.

Methods: When there were massive bleedings in the pelvis, 3 to 5 Foley catheters were inserted through stab wounds on the abdomen. The tips of catheters were placed in the pelvis. Each balloon was inflated with $40 \mathrm{~mL}$ of sterile water. The water inside each balloon was removed gradually from the third postoperative day. If there is no further bleeding, all catheters were removed on the 7 th postoperative day.

Results: There were 8 patients (4 males and 4 females) with massive pelvic bleeding encountered from January 1998 to December 2018. Four patients underwent low anterior resection for primary rectal cancer, 2 patients for colon cancer with pelvic recurrence, 1 patient for ovary cancer with pelvic recurrence, and 1 patient for presacral parachordoma with a bleeding tendency. All of the patients survived without further bleeding after surgery.

Conclusion: The technique is a simple method to control massive pelvic bleeding during colorectal surgery. It is also inexpensive and effective even in patients with a bleeding tendency.

Keywords: Hemostasis; Pelvic bleeding; Massive; Foley catheter

\section{INTRODUCTION}

Hemostasis is a major procedure during any kind of surgery. Although ligation with either absorbable or nonabsorbable sutures has been regarded as the standard for hemostasis, alternative methods such as electrocoagulation, laser evaporation, and harmonic scalpel also are used to stop bleeding [1-3]. However, when there is massive bleeding, especially in the presacral area, packing

Received: Aug 13, 2020 - Revised: Nov 3, 2020 - Accepted: Dec 10, 2020 Correspondence to: Tzu-Chi Hsu, M.D.

Division of Colon and Rectal Surgery, Department of Surgery, Mackay

Memorial Hospital, \#92, Section II, Chung-San North Road, Taipei, Taiwan

Tel: +886-2-2543-3535, Fax: +886-2-2543-3642

E-mail: tzuchi@mmh.org.tw

ORCID: https://orcid.org/0000-0002-0462-1037

- This study was previously presented as a poster at the 14th Annual Meeting of the European Society of Coloproctology in 2019.

(C) 2022 The Korean Society of Coloproctology

This is an open-access article distributed under the terms of the Creative Commons Attribution NonCommercial License (https://creativecommons.org/licenses/by-nc/4.0) which permits unrestricted non-

commercial use, distribution, and reproduction in any medium, provided the original work is properly cited. and occluder pin (Hayden Medical Inc., Santa Clarita, CA, USA) might be necessary to stop such types of bleeding $[4,5]$. The aim of this retrospective clinical study was to describe an alternative method to stop massive bleeding in the pelvis during colorectal surgery.

\section{METHODS}

This study was approved by the Institutional Review Board of Mackay Memorial Hospital in Taipei, Taiwan (No. 18MMHIS106). The written informed consent was obtained for this study and accompanying clinical images.

When there was massive bleeding encountered during the operation, Foley balloons were used to tamponade and stop the bleeding. Three to 5 Foley catheters, number 22 or number 24 in size, were inserted through stab wounds on the abdomen (Fig. 1). The tips of the Foley catheter were placed in the pelvis. Each balloon was inflated with $40 \mathrm{~mL}$ of sterile water to keep the catheter from falling out of the pelvis (Fig. 2). Gross active bleeding would be stopped by balloon compression (Fig. 3). Three Foley catheters 


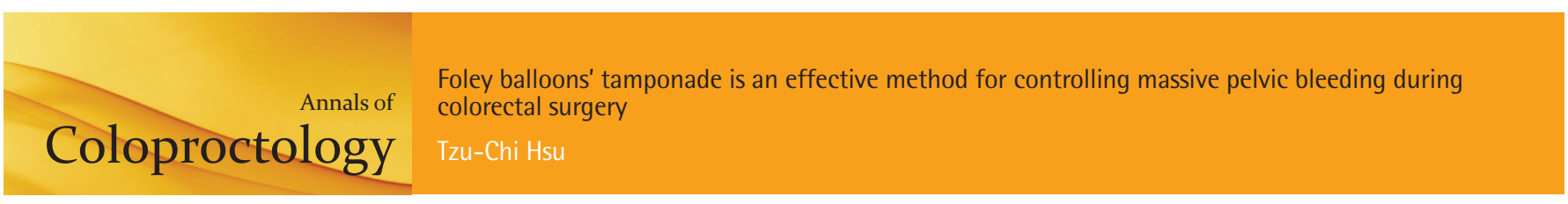

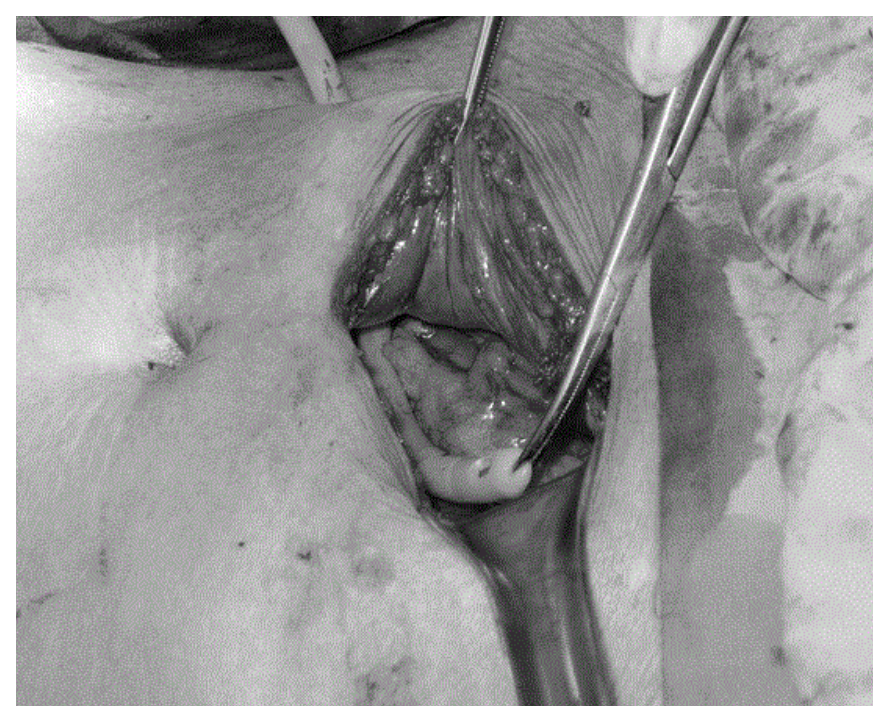

Fig. 1. Insertion of Foley catheter through a stab wound on the abdomen.

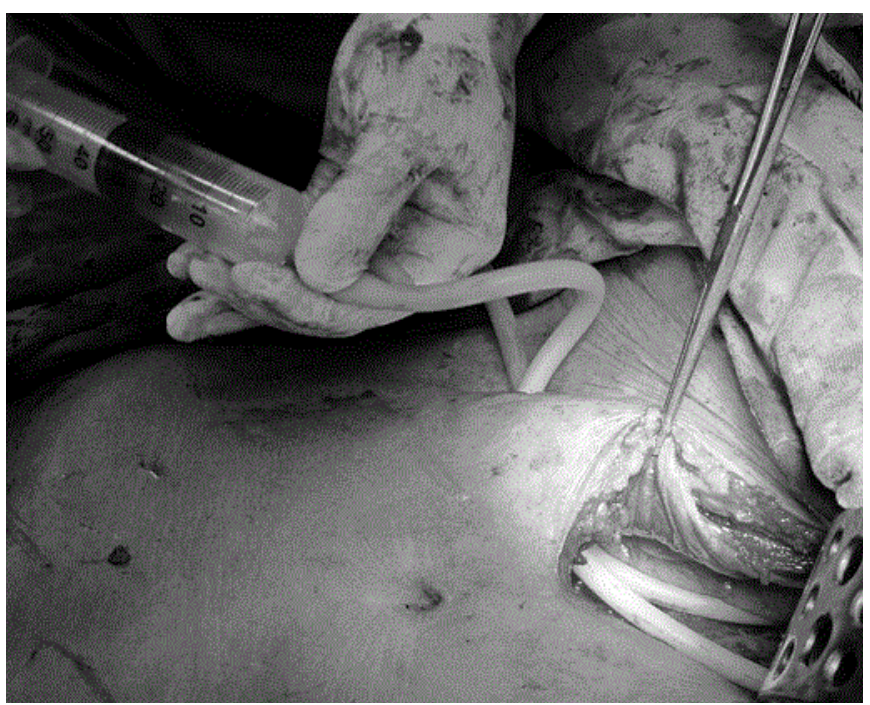

Fig. 2. Each balloon was inflated with $40 \mathrm{~mL}$ of sterile water to keep the catheter from falling out of the pelvis.

were placed in the pelvis for compression and anchored on the abdomen by a few nonabsorbable sutures (Fig. 4). Starting from the third postoperative day, the water inside each balloon was removed gradually, at the pace of $10 \mathrm{~mL}$ each day. If there is no further bleeding, all of the Foley catheters were removed on the 7th postoperative day. We used rubber Foley catheters in the entire series. There was no difficulty encountered upon removal of the Foley catheter at bedside. It is true that usually $10 \mathrm{~mL}$ of water is injected into a fixing balloon. However, it is proven that the balloon can withstand $40 \mathrm{~mL}$ of water. It was tested several times before they were used without rupture of balloon. It is also true that

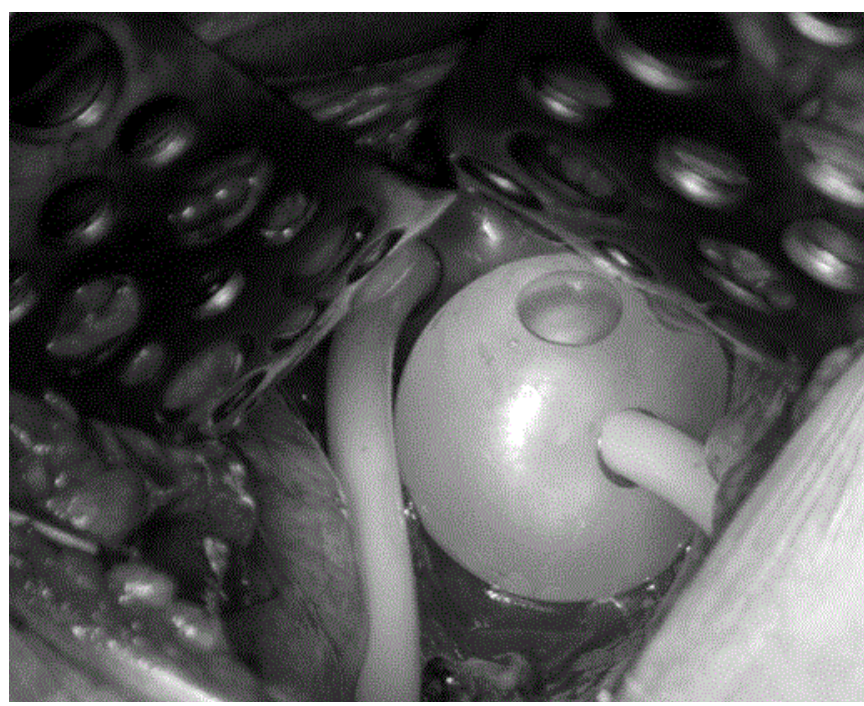

Fig. 3. Gross active bleeding would be stopped by balloon compression.

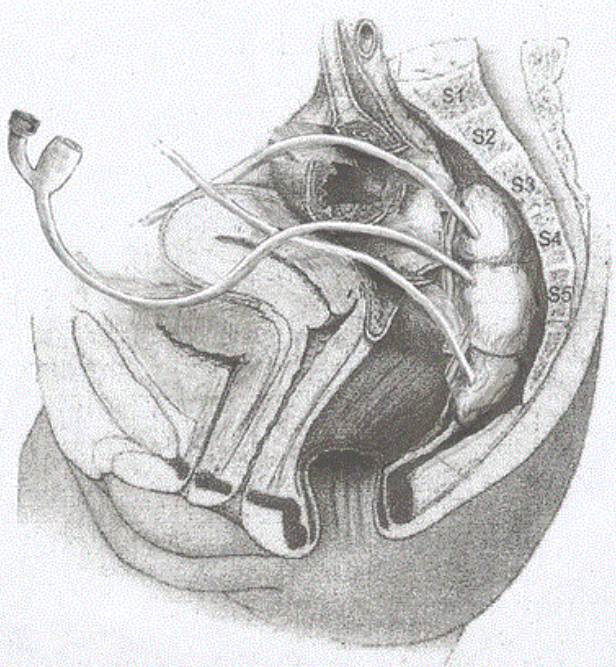

Fig. 4. Three Foley catheters were placed in the pelvis for compression.

Foley catheter might move around. However, with the surrounding bony structure, Foley catheters remained to be stayed in the pelvic cavity in all of the author's cases.

\section{RESULTS}

From January 1998 to June 2020, a total of 8 patients were treated with the technique to stop massive pelvic bleeding. There were 4 males and 4 females. The patients' ages ranged from 35 to 72 years old, with an average of 53 years old. Four patients underwent low anterior resection for primary rectal cancer, 2 patients for colon 
cancer with pelvic recurrence, 1 patient for ovary cancer with pelvic recurrence, and 1 patient for presacral parachordoma with a bleeding tendency. The last patient was a 48-year-old woman, with end-stage renal disease and hemodialysis dependant. She presented with signs of intestinal obstruction. An $11.0 \times 11.0 \times$ $10.5-\mathrm{cm}$ presacral tumor with severe compression on the rectum resulting in rectal stricture was found. A resection of the presacral tumor was difficult due to the large size of the tumor, difficult location of the tumor at the area of hypervascularity, a tendency for high blood loss due to hemodialysis and uremia, and poor physiological status of the patient secondary to uremia. A sigmoid colectomy with anastomosis and resection of the presacral tumor was performed. Severe bleeding was encountered during the operation. Foley balloons' tamponade was used to stop the bleeding. Foley catheters for compression were removed on the 8th postoperative day [6]. All of the patients survived without further bleeding after surgery. Foley catheters were not removed in 1 setting. After 4 days of stable vital sign and hemogram, together without active bleeding from and around the Foley catheters, $10 \mathrm{~mL}$ of water was removed each day. So, it took up to 4 days to have 40 $\mathrm{mL}$ of water removed. There were no complications or failure of bleeding control related to the procedure in the series. Unfortunately, we did not routinely order computed tomography (CT) scan following stopping of bleeding; however, we did have 1 patient who had a pelvic abscess a few months later possibly due to residual blood in the pelvis. We ordered a CT scan for that patient when abscess developed, and the case was reported in the journal of Surgery Today [6].

\section{DISCUSSION}

Although ligation with either absorbable or nonabsorbable sutures has been regarded as a standard for hemostasis, alternative methods such as electrocoagulation, laser evaporation, and harmonic scalpel also are used to stop bleeding [1-3]. As conventional hemostatic measures often fail to control hemorrhage, several alternative methods to control bleeding definitively have been described. When there is massive bleeding, especially in the presacral area, packing with gauze and thumbtacks (hemorrhage occluder pins) might be necessary to stop such kind of massive bleeding. Hemorrhage occluder pins mentioned in the literature showed that it is most effective in presacral venous bleeding and is probably not so effective in other kinds of bleeding. Additionally, an obstacle of occluder pins is that it might be cumbersome while manipulating the instrument in applying pins in some patients. On top of that, it is expensive $[4,5]$. The author proposes an alternative practical comprehensive technique to be used.

It is well-known that Sengstaken-Blakemore tube is effective in controlling bleeding caused by rupture of esophageal varices. Experience of Foley balloon compression for post hemorrhoidectomy bleeding has also been well-documented in the literature [7].
Using the same kind of principle in using a balloon to compress the bleeding site, the author describes a method to stop massive bleeding in the pelvis during colorectal surgery. The method used Foley catheters, sterile water, and syringes, which are readily available in the operation room. There were 8 patients with massive pelvic bleeding treated with this method. There were patients with primary and recurrent cancers, recurrent gynecological cancer, and presacral parachordoma with a bleeding tendency. The last patient was a patient with bleeding tendency due to end-stage renal disease and was hemodialysis dependant. All of the patients survived and without further bleeding after surgery.

Other hemostatic materials such as Floseal Hemostatic Matrix (Baxter, Deerfield, IL, USA) or Surgicel Fibrillar absorbable hemostat (Ethicon, Summerville, NJ, USA) might be helpful for usual bleeding; however, it is not suitable for the pelvic massive bleeding with bleeding loss over $1,000 \mathrm{~mL}$ described in this study. Not to mention the high price of those materials. The technique is a simple method to control massive pelvic bleeding during colorectal surgery. The technique is not only effective and lifesaving but also inexpensive. It is also proven to be a viable option in patients with a bleeding tendency.

In conclusion, the technique is a simple method to control massive pelvic bleeding during colorectal surgery. It is also inexpensive and effective even in patients with a bleeding tendency.

\section{CONFLICT OF INTEREST}

No potential conflict of interest relevant to this article was reported.

\section{REFERENCES}

1. Celentano V, Ausobsky JR, Vowden P. Surgical management of presacral bleeding. Ann R Coll Surg Engl 2014;96:261-5.

2. McCourtney JS, Hussain N, Mackenzie I. Balloon tamponade for control of massive presacral haemorrhage. Br J Surg 1996;83:222.

3. Ng X, Chiou W, Chang S. Controlling a presacral hemorrhage by using a saline bag: report of a case. Dis Colon Rectum 2008;51: 972-4.

4. Stolfi VM, Milsom JW, Lavery IC, Oakley JR, Church JM, Fazio VW. Newly designed occluder pin for presacral hemorrhage. Dis Colon Rectum 1992;35:166-9.

5. Kluger Y, Sagie B, Soffer D, Nasrallha N, Aladgem D. The use of hemorrhage occluder pins for controlling paravertebral intercostal artery bleeding: case report. J Trauma 1997;43:687.

6. Hsu TC, Chen BF, Wang TE. Presacral parachordoma causing intestinal obstruction in a patient with renal failure: report of a case. Surg Today 2011;41:1401-4.

7. Smellie GD. Control of post-haemorrhoidectomy bleeding with a Foley catheter and a pack. J R Coll Surg Edinb 1965;11:328. 